\title{
Renormalizable Massive Vector-Boson Theories with Distorted Local Gauge Symmetry.
}

J. P. HsU

Center for Particle Theory, The University of Texas at Austin - Austin, Tex.

(Lett. Nuovo Cimento, 11, $525(1974))$

Note added. - We assume that each component $\alpha(\alpha=1,2, \ldots)$ of the gauge condition (4) cannot be fixed for all times.

(C) by Società Italiana di Fisica

Proprietà letteraria riservata

Direttore responsabile: CARLO CASTAGNOLI

Stampato in Bologna dalla Tipografla Compositori coi tipi della Tipografia Monograf Questo fascicolo è stato licenziato dai torchi il 27-I-1975 